\title{
Purifying Noisy Entanglement Requires Collective Measurements
}

\author{
N. Linden, ${ }^{1}$ S. Massar, ${ }^{2}$ and S. Popescu ${ }^{1,3}$ \\ ${ }^{1}$ Isaac Newton Institute for Mathematical Sciences, Cambridge, CB3 OEH, United Kingdom \\ ${ }^{2}$ Institute for Theoretical Physics, Princetonplein 5, PO Box 80006, 3508 TA Utrecht, The Netherlands \\ ${ }^{3}$ BRIMS, Hewlett-Packard Laboratories, Stoke Gifford, Bristol, BS12 6QZ, United Kingdom
}

(Received 1 May 1998)

\begin{abstract}
Known entanglement purification protocols for mixed states use collective measurements on several copies of the state in order to increase the entanglement of some of them. We address the question of whether it is possible to purify the entanglement of a state by processing each copy separately. While this is possible for pure states, we show that this is impossible, in general, for mixed states. The importance of this result both conceptually and for experimental realization of purification is discussed. We also give explicit invariants of an entangled state of two qubits under local actions and classical communication. [S0031-9007(98)07322-0]
\end{abstract}

PACS numbers: 03.67.- a, 03.65.Bz

Entanglement is perhaps the key resource which distinguishes quantum from classical information theory. It plays a central role in quantum computation [1] and quantum error correcting codes [2], and it gives rise to some completely new applications such as dense coding [3], teleportation [4], and certain forms of quantum cryptography [5]. In order to function optimally these applications require maximally entangled states. Otherwise the dense coding, teleportation, or quantum cryptography will be imperfect and/or noisy. However, interactions with the environment always occur, and will degrade the quality of the entanglement. If the destructive effects of the environment are not too important, then they can be counteracted by entanglement concentration [6] or entanglement purification [7-9]. This is realized by carrying out local measurements on the entangled particles and classical communication. The entanglement of some pairs is thereby increased at the expense of the entanglement of the others which is destroyed.

There are two fundamentally different types of purification protocols: those acting on individual pairs of entangled particles and those acting collectively on many pairs. In this Letter we address the following question: Is it the case that, whenever it is possible to purify by collective actions, it is also possible to purify by actions on individual pairs?

In the case of pure states this is indeed true. One can always, with finite probability, bring an individual entangled pure state to a maximally entangled state using only local operations [6]. The main result of this Letter is to show that there are situations in which entanglement cannot be purified by actions on individual pairs, even though it can be purified by collective actions. This result is surprising because we expect entanglement to be a property of each pair individually rather than a global property of many pairs.

Specifically, we consider the case of Werner density matrices [10] for two spin- $1 / 2$ particles. It is known [7] that it is always possible to purify singlets from
Werner density matrices by collective methods (if the initial Werner density matrix is entangled). However, we will show that is not possible to purify singlets, or even increase the fidelity of a Werner density matrix infinitesimally, by any combination of local actions and classical communication acting on individual pairs. This is the case even though Werner states do have active nonlocality at the single-pair level, since a single Werner state can realize teleportation (although the teleportation is imperfect, it nonetheless has fidelity better than any classical procedure [11]).

As well as its implications for conceptual aspects of nonlocality, our result has relevance to the experimental realization of purification. The main experimental difficulty, which has so far prevented implementation in the laboratory, is that purification protocols generally require collective measurements on many entangled pairs. Such measurements are very delicate as they involve controlled interactions among different particles. On the other hand, measurements on individual particles are much easier to realize. For instance, photodetectors and polarized beam splitters efficiently realize von Neumann measurements on the polarization of photons. More general positive operator valued measurements (POVM's) which necessitate the use of an ancilla have already been carried out. In the case of photon polarization, the momentum of the photon serves as a convenient ancilla and an arbitrary POVM on a photon can be realized with present technology (see [12] and $[13,14])$.

Thus our result is disappointing from an experimental point of view since it means that purification of arbitrary states cannot be realized using present technology.

We now turn to the proof of our result. We consider Werner states [10], namely, states of two qubits of the form

$$
W(F)=F S+\frac{1-F}{3}\left(1_{4}-S\right),
$$

where $S$ is the projection operator onto the singlet state, $\psi=(\uparrow \downarrow-\downarrow \uparrow) / \sqrt{2}$, and $1_{4}$ is the $4 \times 4$ identity matrix. 
$F=\operatorname{tr}[W(F) S]$ is the fidelity of the Werner state. These states play a central role in purification protocols because, by carrying out suitably chosen unitary transformations on both particles, one can always bring any entangled state to the Werner form. For $F \leq 1 / 2$ a Werner state is unentangled and can be expressed as a mixture of product states. But for $1>F>1 / 2$ there are purification protocols which can extract states with arbitrary large entanglement from an initial set of Werner states. The simplest purification protocol which has been described uses collective measurements on pairs of Werner states [7]. We shall show that it is impossible to increase the fidelity of a Werner state by local operations and classical communication on an individual copy.

Consider a single copy of the mixture $\rho$ of two qubits shared between Alice and Bob (later we will consider the specific case of a Werner state). After carrying out local actions and classical communication they will obtain a density matrix $\rho_{\text {final }}$. In our proof it will be convenient to use the "entanglement of formation" [2] as a measure of the entanglement of $\rho$ and $\rho_{\text {final }}$. It is defined as follows:

(i) For a pure state $|\psi\rangle$ shared between Alice and Bob, $E(\psi)=-\operatorname{tr} \rho_{A} \ln _{2} \rho_{A}=-\operatorname{tr} \rho_{B} \ln _{2} \rho_{B}, \quad$ where $\rho_{A}=$ $\operatorname{tr}_{B}|\psi\rangle\langle\psi|$ and $\rho_{B}=\operatorname{tr}_{A}|\psi\rangle\langle\psi|$.

(ii) For a mixed state $\rho$ the entanglement of formation is the minimum entanglement of the mixtures of pure states that realize $\rho: E(\rho)=\min \sum_{i} p_{i} E\left(\psi_{i}\right)$, where the minimum is taken over all $p_{i}, \psi_{i}$ such that $\rho=$ $\sum_{i} p_{i}\left|\psi_{i}\right\rangle\left\langle\psi_{i}\right|$.

Hill and Wootters have given an explicit formula for the entanglement of formation in the case of two entangled qubits $[15,16]$. They introduce the operation of time reversal $\sim$. For a single qubit the density matrix may be written as $\rho=\frac{1}{2}\left(1_{2}+\alpha \cdot \sigma\right)$ (where $\alpha \cdot \alpha \leq 1,1_{2}$ is the $2 \times 2$ identity matrix, and $\sigma_{i}$ are the Pauli matrices). Then $\tilde{\rho}:=\sigma_{y} \rho^{*} \sigma_{y}=\frac{1}{2}\left(1_{2}-\alpha \cdot \sigma\right)$, where complex conjugation is performed in the basis in which $\sigma_{z}$ is diagonal. For a state of two qubits, the time reversal operation is $\tilde{\rho}=\sigma_{y} \otimes \sigma_{y} \rho^{*} \sigma_{y} \otimes \sigma_{y}$. Now consider the (non-Hermitian, but positive) matrix $\rho \tilde{\rho}$ and denote by $\lambda_{i}$ the positive square root of its eigenvalues:

$$
\rho \tilde{\rho}\left|\tilde{v}_{i}\right\rangle=\lambda_{i}^{2}\left|\tilde{v}_{i}\right\rangle .
$$

The "concurrence" of the state $\rho$ is defined by

$$
C(\rho)=\max \left\{0, \lambda_{1}-\lambda_{2}-\lambda_{3}-\lambda_{4}\right\},
$$

where the $\lambda_{i}$ are taken in decreasing order, and the entanglement of formation $E(\rho)$ is

$$
E(\rho)=H\left(\frac{1+\sqrt{1-C^{2}(\rho)}}{2}\right),
$$

where

$$
H(p)=-p \ln _{2} p-(1-p) \ln _{2}(1-p) .
$$

Note that $0 \leq C \leq 1$ and that $E(C)$ is a strictly monotonic function of $C$ so that the concurrence is a measure of entanglement which is equivalent to the entanglement of formation, i.e., $E\left(\rho_{1}\right)=E\left(\rho_{2}\right)$ if and only if $C\left(\rho_{1}\right)=C\left(\rho_{2}\right)$.

To proceed, we must describe explicitly the possible local operations that can be carried out on a density matrix $\rho$. Then we shall compute how the entanglement of formation changes under these local operations. Consider a mixture $\rho$ of two qubits shared between Alice and Bob. Any purification protocol can be conceived as successive rounds of measurements and communication by Alice and Bob. Suppose Alice carries out the first measurement. It can have many different outcomes. Let us suppose that it has outcome $i_{1}$. Then after the measurement the state of the system becomes $A_{i_{1}} \rho A_{i_{1}}^{\dagger}$, up to normalization, where $A_{i_{1}}$ is an arbitrary operator (in general non-Hermitian) acting on the Hilbert space of Alice's particle $\left(A_{i_{1}}^{\dagger} A_{i_{1}}\right.$ are the elements of the POVM realized by Alice [17]). After communicating the result of her measurement to Bob, he carries out a measurement and obtains outcome $j_{1}$. The state of the system is then $\left[A_{i_{1}} \otimes B_{j_{1}}\left(i_{1}\right)\right] \rho\left[A_{i_{1}}^{\dagger} \otimes B_{j_{1}}^{\dagger}\left(i_{1}\right)\right]$, where $B_{j_{1}}\left(i_{1}\right)$ is an arbitrary operator acting on the Hilbert space of Bob's particle which can depend on the outcome $i_{1}$ of Alice's measurement. Therefore, after $N$ rounds of measurements and communication, the state of the system can always be written as

$$
\rho_{\text {final }}=\frac{A \otimes B \rho A^{\dagger} \otimes B^{\dagger}}{\operatorname{tr}\left(A \otimes B \rho A^{\dagger} \otimes B^{\dagger}\right)},
$$

where $A$ and $B$ are arbitrary operators acting on Alice's and Bob's Hilbert space, respectively. $[A$ denotes the product of the $N$ operators $A_{i_{1}}, \ldots, A_{i_{N}}\left(i_{1}, j_{1}, i_{2}, \ldots, j_{N-1}\right)$ representing the effects of the $N$ measurements carried out by Alice, and similarly for $B$.]

We will need below an explicit expression for $A$ and $B$. To this end, note that we can always write an arbitrary operator $A$ in the form $A=U_{A 2} f_{A} U_{A 1}$, where $U_{A 1}$ and $U_{A 2}$ are unitary operators and $f_{A}=\nu\left(1_{2}+a \sigma_{z}\right)$, with $0 \leq a \leq 1$ and $0<\nu \leq 1 /(1+a)$, is a filtration along the $z$ axis. The upper bound on $\nu$ arises from the fact that, for $f_{A}$ to be physically realizable, its eigenvalues must be between zero and one. The filtration changes the relative weights of the components of the spin along the $+z$ and $-z$ directions. We now write $A=U_{A 2} U_{A 1} U_{A 1}^{\dagger} f_{A}^{a, \mathbf{z}} U_{A 1}=$ $U_{A} f_{A}^{a, \mathbf{n}}$, where $U_{A}=U_{A 2} U_{A 1}$ and $f_{A}^{a, \mathbf{n}}=\nu\left(1_{2}+a \mathbf{n}\right.$. $\sigma)$ and $\mathbf{n}$ is the vector $+z$ rotated by the action of $U_{A 1}$. This is the expression we shall use below.

In addition to carrying out local measurements and communication, Alice and Bob could also randomize the state they obtain. That is they "forget" which operations they carried out and thus obtain a convex combination of different final states $\sum_{i} p_{i} \rho_{\text {final }}^{i}$. However, such randomization can only decrease the entanglement: $E\left(\sum_{i} p_{i} \rho_{\text {final }}^{i}\right) \leq \sum_{i} p_{i} E\left(\rho_{\text {final }}^{i}\right)$, as shown in [2,7]. This is natural since randomization loses information about the state $\rho_{\text {final }}$. For this reason we shall suppose that Alice and Bob do not carry out randomization. 
Having described how $\rho$ changes under local operations, we must describe how $\tilde{\rho}$ changes. We will then be in a position to calculate how the concurrence changes under local operations. Let us first collect some properties of the time reversal operation. (i) If $\rho=\rho_{A} \otimes \rho_{B}$, then $\tilde{\rho}=\tilde{\rho}_{A} \otimes \tilde{\rho}_{B}$. (ii) If $\rho=O \rho^{\prime} O^{\dagger}$, where $O$ is a (possibly non-Hermitian) operator, then $\tilde{\rho}=\tilde{O} \tilde{\rho}^{\prime} \tilde{O}^{\dagger}$. (iii) If $U_{A}=\cos \theta 1_{2}+i \sin \theta \mathbf{q} \cdot \sigma$ is a unitary transformation carried out by Alice, then $\tilde{U}_{A}=U_{A}$. (iv) If $f_{A}^{a, \mathbf{n}}$ is a filtration carried out by Alice, then $\tilde{f}_{A}^{a, \mathbf{n}}=f_{A}^{a,-\mathbf{n}}$.

Therefore since

$$
\rho_{\text {final }}=\frac{U_{A} f_{A}^{a, \mathbf{n}} \otimes U_{B} f_{B}^{b, \mathbf{m}} \rho f_{A}^{a, \mathbf{n}} U_{A}^{\dagger} \otimes f_{B}^{b, \mathbf{m}} U_{B}^{\dagger}}{t(\rho ; a, \mathbf{n} ; b, \mathbf{m})},
$$

with the normalization

$$
t(\rho ; a, \mathbf{n} ; b, \mathbf{m})=\operatorname{tr}\left[f_{A}^{a, \mathbf{n}} f_{A}^{a, \mathbf{n}} \otimes f_{B}^{b, \mathbf{m}} f_{B}^{b, \mathbf{m}} \rho\right],
$$

then

$$
\tilde{\rho}_{\text {final }}=\frac{U_{A} f_{A}^{a,-\mathbf{n}} \otimes U_{B} f_{B}^{b,-\mathbf{m}} \tilde{\rho} f_{A}^{a,-\mathbf{n}} U_{A}^{\dagger} \otimes f_{B}^{b,-\mathbf{m}} U_{B}^{\dagger}}{t(\rho ; a, \mathbf{n} ; b, \mathbf{m})} .
$$

We have defined $f_{B}^{b, \mathbf{m}}=\mu\left(1_{2}+b \mathbf{m} \cdot \boldsymbol{\sigma}\right)$, where $0 \leq$ $b \leq 1$ and $0<\mu \leq 1 /(1+b)$.

Using the fact that $f_{A}^{a, \mathbf{n}} f_{A}^{a,-n}=\nu^{2}\left(1-a^{2}\right) 1_{2}$ and $f_{B}^{b, \mathbf{m}} f_{B}^{b,-\mathbf{m}}=\mu^{2}\left(1-b^{2}\right) 1_{2}$, one finds that

$$
\begin{aligned}
\rho_{\text {final }} \tilde{\boldsymbol{\rho}}_{\text {final }}= & \frac{\mu^{2} \nu^{2}\left(1-a^{2}\right)\left(1-b^{2}\right)}{t^{2}(\rho ; a, \mathbf{n} ; b, \mathbf{m})} U_{A} f_{A}^{a, \mathbf{n}} \\
& \otimes U_{B} f_{B}^{b, \mathbf{m}} \rho \tilde{\rho} f_{A}^{a,-\mathbf{n}} U_{A}^{\dagger} \otimes f_{B}^{b,-\mathbf{m}} U_{B}^{\dagger} .
\end{aligned}
$$

From this expression, one obtains the eigenvalues of $\rho_{\text {final }} \tilde{\rho}_{\text {final }}$ which we need to compute the concurrence of $\rho_{\text {final }}$ :

$$
\rho_{\text {final }} \tilde{\rho}_{\text {final }}\left|\tilde{w}_{i}\right\rangle=\frac{\mu^{4} \nu^{4}\left(1-a^{2}\right)^{2}\left(1-b^{2}\right)^{2}}{t^{2}(\rho ; a, \mathbf{n} ; b, \mathbf{m})} \lambda_{i}^{2}\left|\tilde{w}_{i}\right\rangle,
$$

where

$$
\left|\tilde{w}_{i}\right\rangle=U_{A} f_{A}^{a, \mathbf{n}} \otimes U_{B} f_{B}^{b, \mathbf{m}}\left|\tilde{v}_{i}\right\rangle
$$

and $\left|\tilde{v}_{i}\right\rangle$ is an eigenvector of $\rho \tilde{\rho}$ with eigenvalue $\lambda_{i}^{2}$. Hence

$$
C\left(\rho_{\text {final }}\right)=\frac{\mu^{2} \nu^{2}\left(1-a^{2}\right)\left(1-b^{2}\right)}{t(\rho ; a, \mathbf{n} ; b, \mathbf{m})} C(\rho) .
$$

Since the entanglement of formation is a strictly increasing function of the concurrence $C(\rho)$, the entanglement of formation can only increase if $C$ increases.

To complete the calculation we need the normalization $t$. To this end we introduce the following representation of a density matrix of two qubits

$$
\begin{gathered}
\rho=\frac{1}{4}\left[1_{4}+\boldsymbol{\alpha} \cdot \sigma \otimes 1_{2}+1_{2} \otimes \boldsymbol{\beta} \cdot \sigma\right. \\
\left.+R_{i j} \sigma_{i} \otimes \sigma_{j}\right] .
\end{gathered}
$$

A straightforward calculation then yields

$$
t(\rho ; a, \mathbf{n} ; b, \mathbf{m})=\mu^{2} \nu^{2}\left[\left(1+a^{2}\right)\left(1+b^{2}\right)+2 a\left(1+b^{2}\right) \mathbf{n} \cdot \boldsymbol{\alpha}+2 b\left(1+a^{2}\right) \mathbf{m} \cdot \boldsymbol{\beta}+4 a b R_{i j} n_{i} m_{j}\right] .
$$

For a Werner state $\alpha=\beta=0, R_{i j}=\frac{1-4 F}{3} \delta_{i j}$, and $t(W(F) ; a, \mathbf{n} ; b, \mathbf{m})=\mu^{2} \nu^{2}\left[\left(1+a^{2}\right)\left(1+b^{2}\right)\right.$

$$
\left.+\frac{4}{3}(1-4 F) a b \mathbf{n} \cdot \mathbf{m}\right]
$$

Simple algebra then shows that $C\left(\rho_{\text {final }}\right) \leq C(\rho)$, which proves that the entanglement of formation of a Werner state can never be increased by local operations on a single copy. In fact, it is possible to show that, for any Bell-diagonal state (i.e., one with $\alpha=\beta=0$ ), the entanglement of formation cannot be increased by local actions on an individual copy.

We note that the above result also shows that local operations and classical communication cannot increase the fidelity of an entangled Werner matrix. This is because, for Werner matrices, the entanglement of formation is an increasing function of the fidelity (although $\rho_{\text {final }}$ is not necessarily of Werner form, it can be randomized, and thus brought into Werner form, without increasing its entanglement of formation).
The results that we have described above have been obtained by brute force. However, we would like to understand in a deeper way why density matrices behave differently from pure states as far as entanglement purification is concerned. By actions on a single copy of any entangled pure state one can extract a singlet, with finite probability. Why can the same thing not be achieved for density matrices? We do not know the complete answer to this question yet. However, we can gain some intuition by analyzing the following scenarios.

Consider first the case of a pure state $\psi$ of two spin$1 / 2$ particles. We wish to obtain a singlet from it. This can be achieved [6] but only with a given probability $P$ of success. This probability depends on the initial state $\psi$. Indeed, the overall average amount of entanglement in the system cannot increase, so the initial entanglement $E_{\psi}$ and the probability $P$ must satisfy the inequality

$$
P E_{\text {singlet }} \leq E_{\psi} \text {. }
$$

Thus if we start from different initial states $\psi$, which are closer and closer to a nonentangled state, one finds that although one can always obtain a singlet, the probability of success must, and indeed does, go to zero. 
Now suppose it were the case that purifying density matrices can be achieved in a similar way, namely that a given goal (a given final state) can always be obtained from any initial state $\rho$ with some nonzero probability of success. Specifically, let our goal be to obtain a fixed Werner state with fidelity $F_{\text {final }}>1 / 2$ (it may be too ambitious to try to obtain a singlet, so we do not assume that $F_{\text {final }}=1$ ) and let us assume that for any initial fidelity $1 / 2<F<F_{\text {final }}$ this can be done. Once again if we consider what happens as our initial state tends towards the unentangled Werner state (with $F=1 / 2$ ), the probability of success must tend towards zero. However, as we show below, it turns out that, no matter what local actions we perform, any possible outcome of the measurement occurs with a finite probability which does not tend to zero as $F \rightarrow 1 / 2$. Roughly speaking, this is because the nonentangled limit of the family of Werner states, namely, the Werner state with $F=1 / 2$, is still a mixed state, and the noise contained in it does not allow any outcome of any measurement to remain "silent." Thus there can be no measurement which could achieve the goal described.

Consider $W_{\text {final }}$ to be our fixed goal. Suppose that it were possible to choose actions which allowed one to obtain $W_{\text {final }}$ starting from $W(F)$. Then $W_{\text {final }}$ would be given by

$$
W_{\text {final }}=\frac{U_{A} f_{A}^{a, \mathbf{n}} \otimes U_{B} f_{B}^{b, \mathbf{m}} W(F) f_{A}^{a, \mathbf{n}} U_{A}^{\dagger} \otimes f_{B}^{b, \mathbf{m}} U_{B}^{\dagger}}{t(W(F) ; a, \mathbf{n} ; b, \mathbf{m})},
$$

as in (6). The probability of obtaining $W_{\text {final }}$ would then be equal to the normalization $t(W(F) ; a, \mathbf{n} ; b, \mathbf{m})$ which is given in (15). It is straightforward to show that this probability does not go to zero as $F \rightarrow 1 / 2$ (excluding the trivial case $\mu$ or $\nu$ equal to zero, in which one filters out all of the particles). Thus one cannot purify to a fixed output state.

The above argument just shows that mixed states cannot have the same simple behavior as pure states. More subtle behavior is not ruled out by the argument. For example it might have been the case that an individual Werner state can be purified only in a small range of fidelities, say $F_{\min }<F<F_{\text {final }}$, with $F_{\min }>1 / 2$. If this were the case, then as $F \rightarrow 1 / 2$ the probability of obtaining $W_{\text {final }}$ need not tend to zero since as $F \rightarrow 1 / 2$ the state cannot be purified. The proof given in the first part of this Letter, however, shows that this is not the case.

Finally, we note that our expression for the eigenvalues of $\rho \tilde{\rho}$ shows that their ratios $\lambda_{i}^{2} / \lambda_{j}^{2}$ are invariant under arbitrary local operations and classical communication (excluding randomizations). This therefore provides a characterization of the equivalence classes of density matrices under such operations. This may have important applications because it provides a simple criterion for distinguishing states whose entanglement is fundamentally different. Whether this characterization is complete, i.e., whether their are additional independent functions of $\rho$ which are invariant under local operations and classical communication, is still an open question. Also how to characterize the equivalence of density matrices under local operations, classical communication, and randomization is unknown. (The invariants of multiparticle entangled states under local unitary operations have been discussed in $[18,19]$.)

We are very grateful to Colin Sparrow for his help at an early stage in this work. S.P. warmly acknowledges very useful discussions with P. K. Aravind.

[1] A. Barenco, Contemp. Phys. 37, 375 (1996).

[2] C.H. Bennett, D.P. DiVincenzo, J.A. Smolin, and W. K. Wootters, Phys. Rev. A 54, 3824 (1996).

[3] C. H. Bennett and S. J. Wiesner, Phys. Rev. Lett. 69, 2881 (1992).

[4] C. H. Bennett, G. Brassard, C. Crépeau, R. Jozsa, A. Peres, and W. K. Wootters, Phys. Rev. Lett. 70, 1895 (1993).

[5] A. K. Ekert, Phys. Rev. Lett. 67, 661 (1991).

[6] C.H. Bennett, H. Bernstein, S. Popescu, and B. Schumacher, Phys. Rev. A 53, 2046 (1996).

[7] C. H. Bennett, G. Brassard, S. Popescu, B. Schumacher, J. A. Smolin, and W. K. Wootters, Phys. Rev. Lett. 76, 722 (1996).

[8] N. Gisin, Phys. Lett. A 210, 151 (1996).

[9] M. Horodecki, P. Horodecki, and R. Horodecki, Phys. Rev. Lett. 78, 574 (1997).

[10] R. F. Werner, Phys. Rev. A 40, 4277 (1989).

[11] S. Popescu, Phys. Rev. Lett. 72, 797 (1994).

[12] B. Huttner, A. Muller, J.D. Gautier, H. Zbinder, and N. Gisin, Phys. Rev. A 54, 3783 (1996).

[13] S. Popescu, quant-ph/9501020.

[14] D. Boschi, S. Branca, F. De Martini, L. Hardy, and S. Popescu, Phys. Rev. Lett. 80, 1121 (1998).

[15] S. Hill and W. K. Wootters, Phys. Rev. Lett. 78, 5022 (1997).

[16] W. K. Wootters, Phys. Rev. Lett. 80, 2245 (1998).

[17] See, e.g., H. Barnum, M. A. Nielsen, and B. Schumacher, Phys. Rev. A 57, 4153 (1998).

[18] N. Linden and S. Popescu, Fortschr. Phys. 46, 567 (1998).

[19] N. Linden, S. Popescu, and A. Sudbery, quant-ph/ 9801076. 